\title{
ELECTRICAL PROPERTIES OF THE FROG SKELETAL MUSCLE MEMBRANE IN Cl-FREE SULPHATE-, FERROCYANIDE-, AND GLUTAMATE- RINGER'S SOLUTIONS
}

\author{
Teruaki Szaimi And Tadao Tomita* \\ Department of Physiology, Faculty of Medicine, \\ Kyushu University, Fukuoka
}

FALK and LANDA (1960a) observed a prolonged response of the skeletal muscle membrane in the absence of penetrating anions. They also observed two different distributions of the value of the resting potential, one of which showed the peak at -95 to $-105 \mathrm{mV}$, and the other, at -35 to $-45 \mathrm{mV}$, in $\mathrm{K}$ - and $\mathrm{Cl}$-free solution. The low resting potential which had a peak at -35 to $-45 \mathrm{mV}$ in distribution seemed to correspond to a plateau level of the prolonged response (FALK and LANDA, 1960b).

In the present experiment, the electrical properties of the frog skeletal muscle fiber having the low resting potential in $\mathrm{Cl}$-free sulphate-, ferrocyanide-, glutamate-Ringer's solutions were mainly investigated. The membrane in such condition showed the similar response to that observed by ADRIAN (1960) in different condition in which the muscle was bathed in $100 \mathrm{mM}-\mathrm{K}$ sulphate solution after it had been loaded with $\mathrm{KCl}$ in $100 \mathrm{mM}-\mathrm{K} 220 \mathrm{mM}-\mathrm{Cl}$ solution. The effect of $\mathrm{K}$ on the electrical properties of the fiber in $\mathrm{Cl}$-free solution was also studied.

\section{METHOD}

Isolated sartorius muscles of a frog (Rana nigromaculata nigromaculata) were mounted in the lucite chamber filled with the test solution, the capacity of which was about $10 \mathrm{cc}$. Two glass capillary microelectrodes were very closely inserted into a single muscle fiber: one was for passing current through the membrane and the other, for recording potential. The current was recorded by the use of a DC amplifier (imput impedance: $20 \mathrm{~K} \Omega$ ) connected between the bath and the ground. The maximum current intensity of $2 \times 10^{-7} \mathrm{~A}$ was used in a series of experiments and therefore the potential difference of $4 \mathrm{mV}$ was produced at the monitoring resistor. To prevent this potential from mixing with the potential produced at the membrane, the differential amplifier was used for recording the membrane potential. The applied

Received for publication July 17, 1963.

* 細美照明, 富田忠雄 
current was passed through the resistor of $100 \mathrm{M} \Omega$ connected in series with the microelectrode.

The solutions used for the test had the following compositions $(\mathrm{mM})$.

Normal Ringer's solution: $\mathrm{NaCl}, 115 ; \mathrm{KCl}, 2.5 ; \mathrm{CaCl}_{2}, 1.8 ; \mathrm{NaHCO}_{3}, 2.4$

Sulphate-Ringer's solution: $\mathrm{Na}_{2} \mathrm{SO}_{4}, 71.99 ; \mathrm{K}_{2} \mathrm{SO}_{4}, 1.56 ; \mathrm{CaSO}_{4}, 8 ; \mathrm{NaHCO}_{3}, 2.4$; sucrose, 52.55

Ferrocyanide-Ringer's solution: $\mathrm{Na}_{4} \mathrm{Fe}(\mathrm{CN})_{6}, 45 ; \mathrm{K}_{4} \mathrm{Fe}(\mathrm{CN})_{6}, 0.95 ; \mathrm{Ca}_{2} \mathrm{Fe}(\mathrm{CN})_{6}, 1.16$; sucrose, 75

Glutamate-Ringer's solution: Na glutamate, $115 ; \mathrm{K}$ glutamate, $2.5 ; \mathrm{CaCl}_{2}, 1.8 ; \mathrm{NaHCO}_{3}$, 2.4

When K-free solution was used, $\mathrm{K}$ was simply omitted out of each solution. When K-excess solution was used, $\mathrm{Na}$ was replaced with an equimolar amount of $\mathrm{K}$. Sulphate- and ferrocyanide-Ringer's solutions had almost the same conductivity as Ringer's solution but glutamate-Ringer's solution had a lower conductivity than Ringer's solution (about half conductivity of Ringer's solution). When the solution was changed, the chamber was slowly irrigated with the test solution of $100 \mathrm{cc}$. The experiment was carried out at room temperature $\left(23-27^{\circ} \mathrm{C}\right)$.

\section{RESULTS}

In Cl-free solutions with normal $K$ concentration. When $\mathrm{Cl}$-ion in Ringer's solution was replaced with sulphate, ferrocyanide or glutamate, fibrillation of the muscle was always observed in each case. Generally the fibrillation became weak gradually and in some fibers it disappeared within ten minutes. In about 20-60 minutes almost all fibers ceased to fibrillate. There were, however, some muscles in which the fibrillation lasted over one hour. The time required for suspension of fibrillation was usually shorter in the order of glutamate-, ferrocyanide- and sulphate-Ringer's solutions.

In general, the electrical properties of the membrane were investigated with the fiber whose fibrillation was ceased, but the insertion of microelectrodes often induced fibrillation, which made the experiment difficult. Though the value of the resting potential of individual fibers showed a large dispersion in $\mathrm{Cl}$-free solutions, it could be divided into two groups: one showed the peak in distribution at about $-80(-50--90) \mathrm{mV}$ (the resting potential of this range is called the high resting potential, H. R.P., hereafter) and the other, the peak in distribution at about $-30(-10--40) \mathrm{mV}$ (the low resting potential, L. R. P.). The values of both H. R. P. and L. R. P. were gradually increased and the former approached the normal resting potential (about $-90 \mathrm{mV}$ ) and the fibers with H.R.P. had a tendency to increase in number in the course of time that the muscle had been in the $\mathrm{Cl}$-free solution. Sometimes, shift from H. R. P. and L. R. P. was observed after insertion of microelectrodes or after electrical stimulations. This shift was always accompanied with a burst of spike potential and fibrillation by which microelectrodes were usually withdrawn. As for the ratio of the fibers which had L. R.P. and H. R. P., there was 
a large difference depending on preparations and solutions used. L. R. P. was observed most in glutamate-, less in ferrocyanide- and least in sulphate-Ringer's solutions.

If recovery of the resting potential was due to decrease of internal $\mathrm{Cl}$ concentration, the very large resting potential would be expected in the fiber which was transferred into K-free Cl-Ringer's solution after soaking in Cl-free Ringer's solution for more than 1 hour until the resting potential became nearly normal. The fact was that the resting potential became higher by $20-25 \mathrm{mV}(100-110 \mathrm{mV}$ in absolute value). This value showed little difference from what was obtained in the fiber transferred from normal Ringer's solution to K-free Cl-Ringer's solution. When the membrane potential was hyperpolarized with such treatment, the threshold was high because the critical level was not changed. These were just similar as in case in which the membrane was hyperpolarized by the conditioning inward current.

FIG. 1 shows the membrane potential produced by the current pulse in sulphate-Ringer's solution. In FIG. 2 and 3 the results in ferrocyanide- and glutamate-Ringer's solutions are shown respectively. FIG. 4 shows the voltagecurrent relation in sulphate-Ringer's solution which was obtained from the records of FIG. 1. As seen in the figures, general characteristics of the voltage-

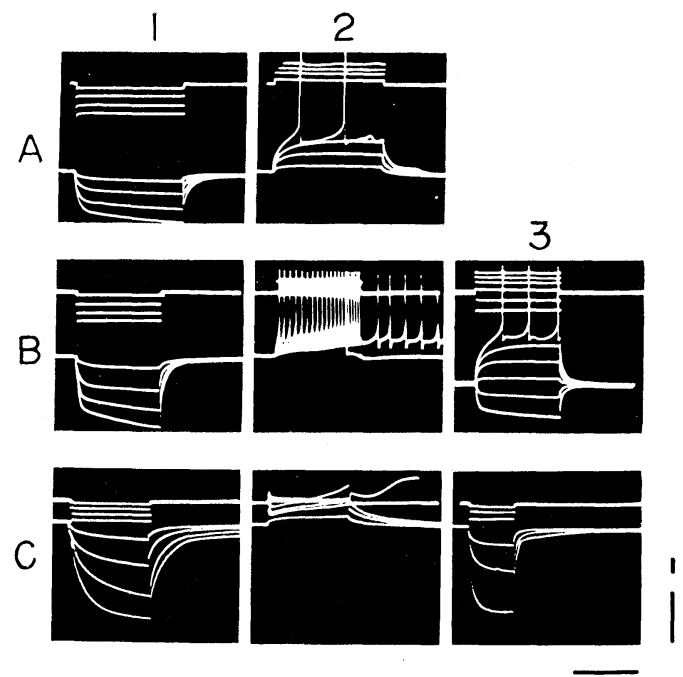

FIG. 1. A : Electrical responses (lower traces) of the muscle membrane produced by square current pulse (upper traces) in normal Ringer's solution. B, C: The responses obtained in sulphate-Ringer's solution. In B-3, the conditioning hyperpolarization was applied; in $\mathrm{C}-2$, movement of the fiber was induced at the biggest depolarization. The base lines of current records correspond to zero membrane potential. Current calibration (upper vertical line) : $10^{-7} \mathrm{~A}$; voltage calibration (lower vertical line) $: 50 \mathrm{mV}$; time scale (horizontal line): $500 \mathrm{msec}$ (100 $\mathrm{msec}$ for A, $2 \mathrm{sec}$ for C-3). 

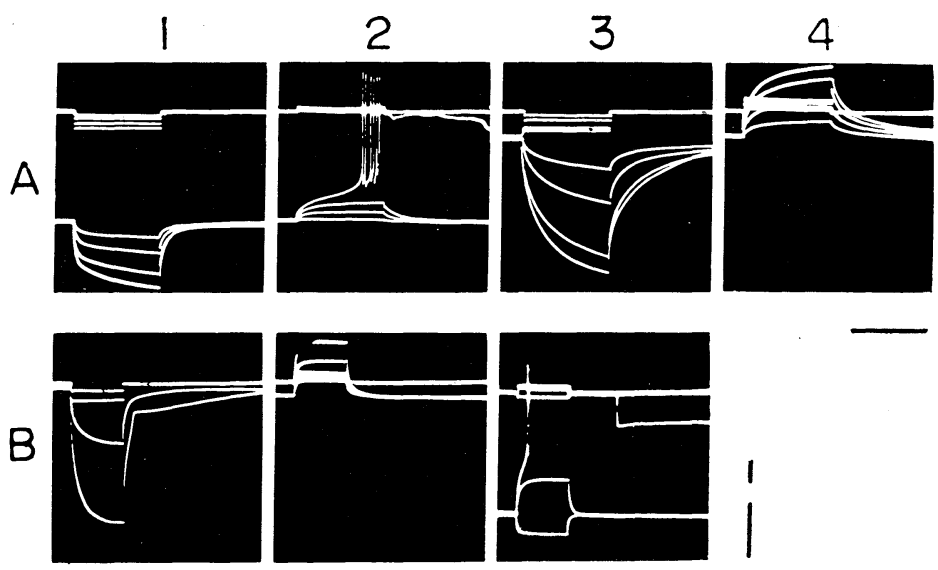

FIG. 2. Records in ferrocyanide-Ringer's solution. In B-3, the conditioning hyperpolarization was applied, and the electrode was once withdrawn after a burst of spike potentials then spontaneously inserted again. Current calibration (upper vertical line): $10^{-7} \mathrm{~A}$; voltage calibration (lower vertical line): $50 \mathrm{mV}$; time scale (horizontal line): $500 \mathrm{msec}$ for $A, 2 \mathrm{sec}$ for $B$.

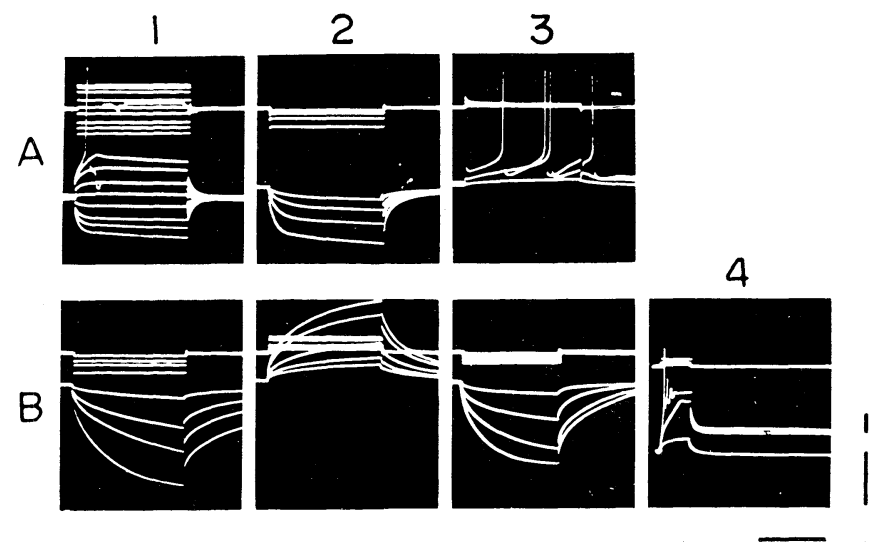

FIG. 3. A-1: Record in normal Ringer's solution. A-2, 3 and B: Records in glutamate-Ringer's solution. In B-4, the conditioning hyperpolarization was applied. Current calibration (upper bar): 10-7 A; Voltage calibration (lower bar): $50 \mathrm{mV}$; time scale (horizontal bar): $100 \mathrm{msec}$ (500 msec for B-3 and 4).

current relation were all the same in sulphate-, ferrocyanide- and glutamateRinger's solutions. The fiber with H. R. P. produced the action potential with both the normal amplitude and duration. The response was, however, always observed in the repetitive form. The electrodes were usually withdrawn due to the movement of the muscle fiber. The threshold was a little low due to 


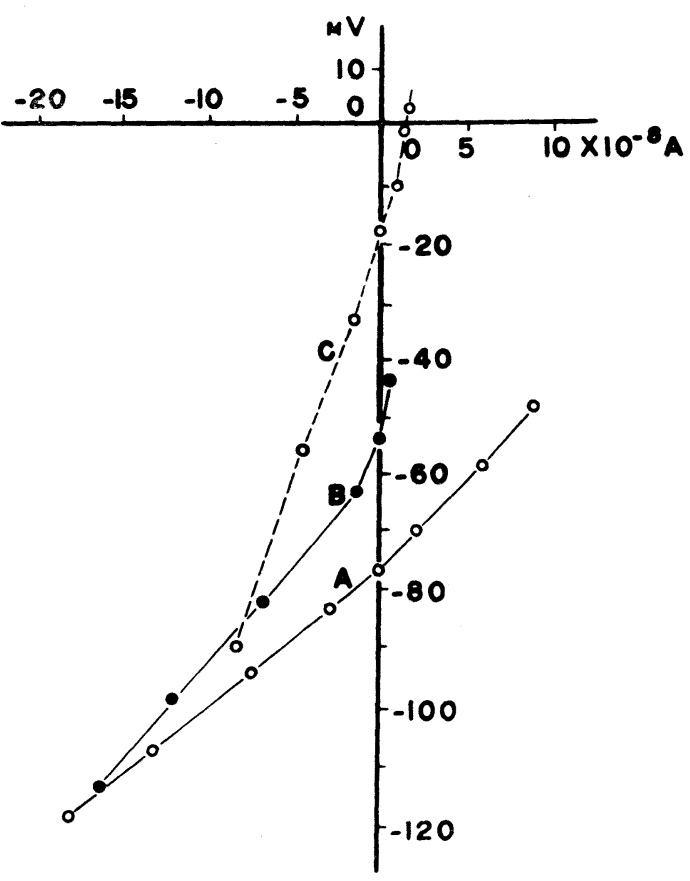

FIG. 4. Current-voltage relations of the muscle fiber bathed in sulphateRinger's solution. Lines $\mathrm{A}, \mathrm{B}$, and $\mathrm{C}$ correspond to records $\mathrm{A}, \mathrm{B}$, and $\mathrm{C}$ in FIG. 1 respectively. Ordinate, membrane potential at the end of the pulses. Abscissa, intensity of the current pulses. Inward current, negative.

the low resting potential. There was little accommodation and the maximum latency became very long. The effective membrane resistance was fairly higher and consequently the membrane time constant was longer than that in Ringer's solution. In each solution, muscles maintained their excitability for many hours. The properties of the muscle fiber completely returned to normal state in a few minutes when the solution was replaced with normal Ringer's solution.

In the fibers with L. R. P., the action potential was not produced (FIG. 1, C; FIg. 2, A-3, 4; Fig. 3, B). The contraction was not usually observed, but in some fibers the contraction was produced by the outward current pulse. The effective membrane resistance became much higher than in the fiber with H. R.P. (FIG. 4). The membrane time constant became so large that steady maximum deflection was not enough to be obtained by the current pulse of about 1 second in duration. After the membrane potential was restored approximately to the normal resting potential level by the conditioning inward current, the membrane was able to have the same response as the fiber with H. R. P. by the outward current pulse (FIG. 2, B-3; FIG. 3, B-4). 
The behavior of the membrane of each fiber with H.R.P. or L. R.P. was essentially identical in sulphate-, ferrocyanide- or glutamate-Ringer's solution respectively. In the fiber with H.R.P., the time course of big hyperpolarization was different from the time course expected from the exponential or error functions. The electrotonic potential was rapidly increased at the start of the current pulse, then the potential was slowly increased when the potential level was over the normal resting potential (about $-90 \mathrm{mV}$ ). Even in the fiber with L. R. P., if the membrane potential was hyperpolarized beyond -90 $\mathrm{mV}$, the similar form of the electrotonic potential to that in the fiber with H. R. P. was found, though the former was not so remarkable as the latter. When the membrane potential was hyperpolarized beyond $-150 \mathrm{mV}$, the electrotonic potential was rather decreased in the course of time and sometimes an irregular fluctuation was observed in the potential.

In the fiber with H.R.P., the response produced by the depolarization made it unable to observe the membrane resistance beyond the critical level. The membrane resistance was increased at the depolarization up to subthreshold potential and slightly decreased at the hyperpolarization. In the fiber with L.R.P. little rectification was observed. When the electrotonic potential became large and approached the normal resting potential, the membrane resistance tended to be decreased. This was remarkable in the fiber with H. R. P.

In the fiber with L. R. P., the time course of the falling phase of the large electrotonic potential was different from that of the rising phase if duration of the current pulse was long enough (over $500 \mathrm{msec}$ ). The longer the pulse duration was and the larger the depolarization potential was, the clearer the difference between the rising and the falling phases became. This difference was clearly caused by some response. As is seen in FIG. 1, C-3 and FIG. 2, B-1 the membrane potential returned to its original level by forming a step after cessation of an inward current pulse. Usually the membrane was rapidly
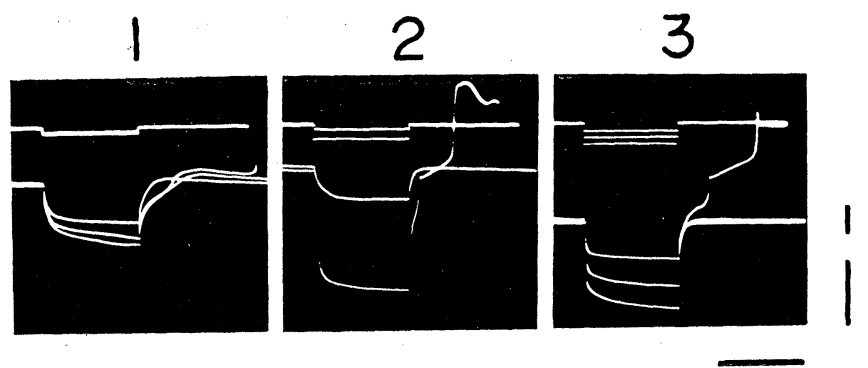

FIG. 5. Records in sulphate-Ringer's solution. No conditioning current was applied. In records 2 and 3 , the anode break response produced movement of the fiber. Calibration: $10^{-7} \mathrm{~A}$ (upper bar), $50 \mathrm{mV}$ (lower bar) and $2 \mathrm{sec}$ (horizontal bar). 

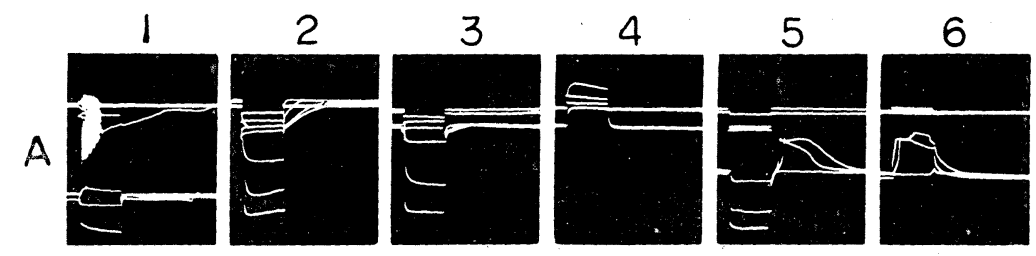

B
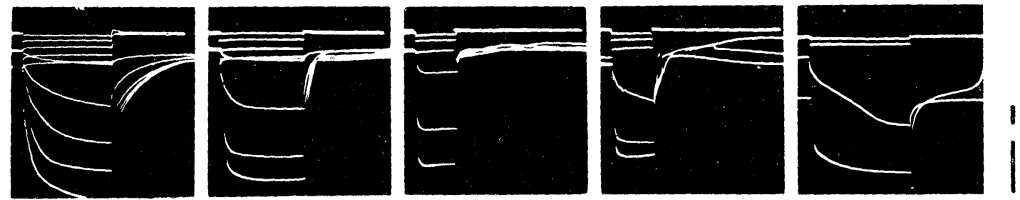

Fig. 6. Records in ferrocyanide-Ringer's solution. Records in row A were obtained from the same fiber. A-3 to A-6: With the conditioning hyperpolarization. Calibration: $10^{-7} \mathrm{~A}$ (upper bar), $50 \mathrm{mV}$ (lower bar). Time scale (horizontal bar): $2 \mathrm{sec}$ (100 $\mathrm{msec}$ for B-1; $500 \mathrm{msec}$ for B-2 and 5).
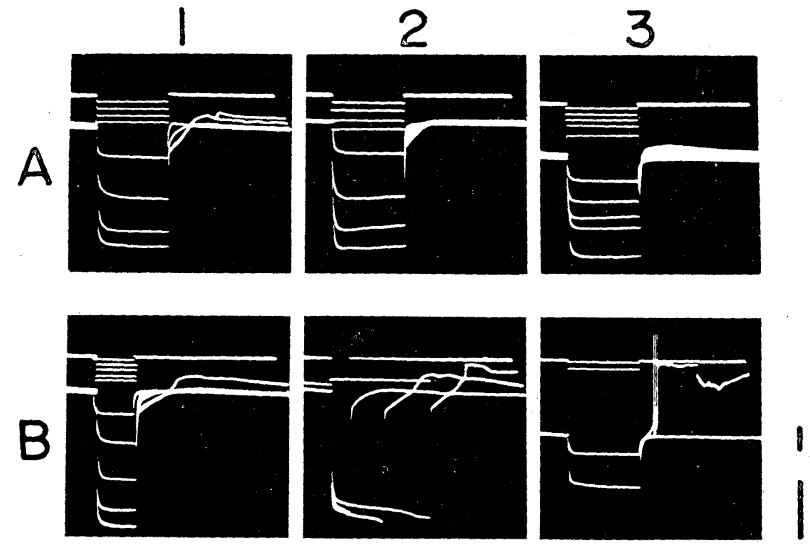

FIG. 7. Records in glutamate-Ringer's solution. Records in row A were obtained from the same fiber. A-3: With the conditioning hyperpolarization. Calibration: $10^{-7} \mathrm{~A}$ (upper bar), $50 \mathrm{mV}$ (lower bar), $2 \mathrm{sec}$ (horizontal bar).

depolarized up to $-30--40 \mathrm{mV}$, afterwards very slowly returned to the original potential level. Sometimes it was further depolarized (inside more positive) beyond the original level and in this case, it was often accompanied with movement of the muscle fiber (FIG. 5 and 7 ).

In the fiber with H.R.P., the shift of the membrane potential to L.R.P. was sometimes observed due to the anode break response (FIG. 6, B-5). When the resting potential was sufficiently large, a burst of the spike potential was observed at the start of the shift (FIG. 7, B-3). L. R. P. was rarely shifted to 
H. R.P. by the inward current pulse. The process in this case was similar as in the hyperpolarizing response observed at the nerve membrane in K-rich solution (FIG. 6, B-5).

The time course of the membrane potential after termination of the current pulse was very changeable depending on the membrane potential levels. In the fully depolarized membrane (with or without conditioning outward current), the response never overshot the original level (FIG. 6, A-2 and FIG. 7, A-2). The membrane potential being a little higher (with or without conditioning inward current), the positive component (overshoot) became more remarkable instead of the negative component (FIG. 7, A-3) and sometimes the prolonged response (FIG. 6, A-5 and 6) was produced in all-or-none manner as observed by NAKAJIMA, IWASAKI and OBATA (1962).

The fiber with L.R.P. was increased in number in K-free ferrocyanideRinger's solution as shown by FALK and LANDA (1960b). The value of L. R.P. became small, while the value of H.R.P. became large. As the result, the difference between them became distinct and the resting potential of - 30 $-70 \mathrm{mV}$ was hardly observed. The same phenomenon was observed not only in ferrocyanide-Ringer's solution but also in sulphate- or glutamate-Ringer's solution. The properties of the fiber in $\mathrm{K}$-free $\mathrm{Cl}$-free solution were essentially the same as that in the solution with the normal $K$ concentration, except the difference in the resting potential.

In $\mathrm{K}$-rich $\mathrm{Cl}$-free solutions. The membrane was depolarized in $\mathrm{K}$-rich $\mathrm{Cl}$-free solution in proportion to $\mathrm{K}$ concentration. These values approximately showed the logarithmic relation with a slope of $58 \mathrm{mV}$ for 10 times as much as $\mathrm{K}$. The resting potential for $\mathrm{K}$ of above $60 \mathrm{mM}$ was, however, observed to be a little larger (inside was more negative) than the predicted value. In $\mathrm{Cl}$-free solution with normal $\mathrm{K}$ concentration, the value of the resting potential of individual fibers showed a large dispersion, while in $\mathrm{K}$-excess solution it was less scattered (within $10 \mathrm{mV}$ ) though it was not observed between $2.5-10 \mathrm{mM} \mathrm{K}$.

FIG. $8 \mathrm{~A}$ shows the potential responses produced by the current pulse in $\mathrm{K}$-rich glutamate solution, and FIG. $8 \mathrm{~B}$ shows the current-voltage relation in graph. As shown in these figures, in $\mathrm{K}$-rich $\mathrm{Cl}$-free solution the anomalous rectification was clearly observed. The membrane resistance was low at the hyperpolarization and high at the depolarization. When the inside of the membrane was polarized to $+60-+100 \mathrm{mV}$, the membrane resistance began to be decreased again, and at the large hyperpolarization (near to the normal resting potential) the resistance tended to be slightly increased. Though the membrane resistance became lower at the hyperpolarization as $\mathrm{K}$ concentration was increased, the configuration of the rectification had no remarkable difference at $\mathrm{K}$ concentrations between $12 \mathrm{mM}$ and $120 \mathrm{mM}$. External $\mathrm{K}$ being increased, the membrane resistance at the depolarization was little decreased, 

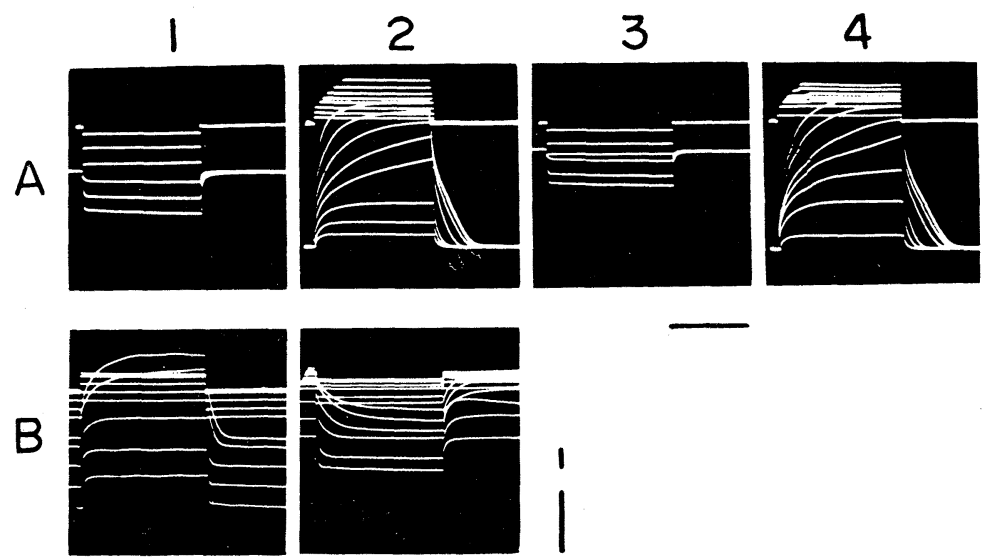

FIG. 8A. Records in K-rich glutamate-Ringer's solution. A-1 and 2; B-1 and 2: $30 \mathrm{mM} \mathrm{K} . \mathrm{A}-3$ and $4: 60 \mathrm{mM} \mathrm{K}$. B-1: With the conditioning hyperpolarization. B-2: With the conditioning depolarization. Calibration: $10^{-7} \mathrm{~A}$ (upper bar), $50 \mathrm{mV}$ (lower bar), $500 \mathrm{msec}$ (horizontal bar).

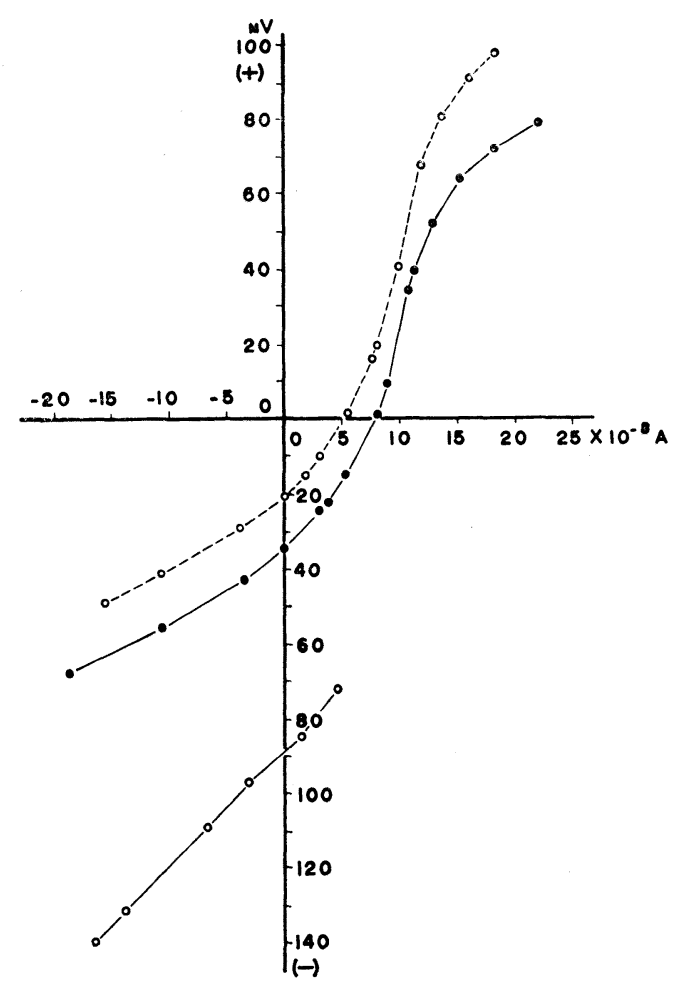

FIG. 8B. Current-voltage relations obtained from the same fiber in FIG. 8A. The uppermost line: in $60 \mathrm{mM}$ glutamate-Ringer's solution; the middle line: in $30 \mathrm{mM} \mathrm{K}$ solution. The lowest line (resting potential, $89 \mathrm{mV}$ ) shows current-voltage relation in normal Ringer's solution. 
and it was nearly the same as in the solution with normal $\mathrm{K}$ concentration.

The membrane resistance was increased with time at the depolarization over $20 \mathrm{mV}$ and electrotonic potential did not become steady by about 1 second pulse duration. However, this tendency became weak at the depolarization above $+70 \mathrm{mV}$ and the electrotonic potential came to reach the steady level quickly. When such a phenomenon appeared at depolarization, the time course of the falling phase of the electrotonic potential was different from that of the rising phase. The falling phase showed the similar time course as observed in the nerve membrane a little hyperpolarized by the conditioning current in K-rich solution (FIG. 8, A-2 and 4). However, this process had a different characteristic from that of a nerve membrane in K-rich solution: it became less prominent when the membrane was hyperpolarized by the conditioning inward current (FIG. 8, B-1).

Anodal pulses applied to the depolarized membrane in K-rich media is known to produce hyperpolarizing response in various excitable tissues (SEGAL, 1958; Stämpfli, 1958; TASAKI, 1959; Koketsu and Koyama, 1962). However, the frog skeletal muscle fiber did not show any marked hyperpolarizing response as seen in nerve fibers.

The strong and long current pulse being applied to the membrane in $\mathrm{K}$ rich $\mathrm{Cl}$-free solution, the internal potential became more positive than the resting level before returning to it, after cessation of the pulse. This slow process was considered as essentially the same as that obtained in the solution with normal $\mathrm{K}$ concentration, and also the slow response observed by ADRIAN (1960). ADRIAN observed that the muscle loaded with $\mathrm{KCl}$ in the solution containing $100 \mathrm{mM} \mathrm{K}, 200 \mathrm{mM} \mathrm{Cl}$ had an internal potential of about $+40 \mathrm{mV}$ and showed slow response by inward current pulse when the external $\mathrm{Cl}$ was replaced with sulphate. FIG. 9 and 10 show the examples obtained in K-rich ferrocyanide- and sulphate-Ringer's solutions. The membrane resistance tended to be increased in the course of time at the membrane potential over some $-70 \mathrm{mV}$. When the membrane was hyperpolarized at such level, the slow break response was observed after the anodal pulse was discontinued. The amplitude of this response increased in graded manner, as the intensity of the current pulse was increased, or as its duration was lengthened. Sometimes the response with different time course from the graded response was produced in all-or-none manner if the pulse was over a certain critical intensity (FIG. 9, A-1, 2 and FIG. 10, B-1). Besides, this response was sometimes accompanied with movement of muscle fiber.

The slow break response became difficult to be produced when the membrane was hyperpolarized by the conditioning inward current (FIG. 9, A-3, B-3 and FIG. 10, B-2, 3). On the other hand, when the membrane was depolarized by the outward current, it became easy to be produced. FIG. 10, C shows the example that the conditioning depolarization made the slow de- 

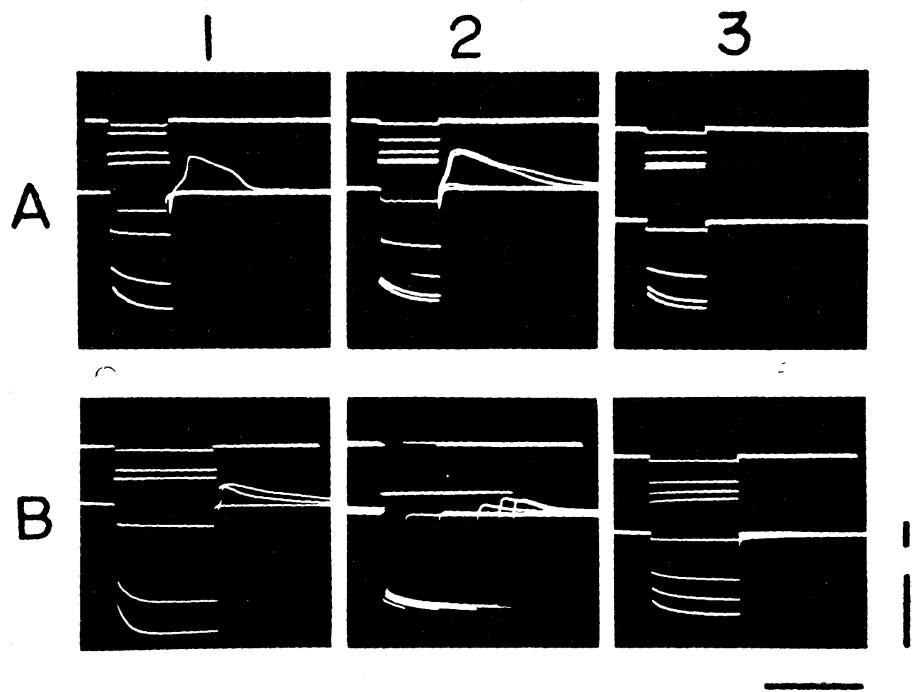

FIG. 9. A: Records in $12 \mathrm{mM} \mathrm{K}$ ferrocyanide-Ringer's solution. B: Records in $20 \mathrm{mM} K$ sulphate-Ringer's solution. $A-3$ and $B-3$ : With the conditioning hyperpolarization. Calibration: $10^{-7} \mathrm{~A}$ (upper bar), $50 \mathrm{mV}$ (lower bar), $2 \mathrm{sec}$ (horizontal bar).

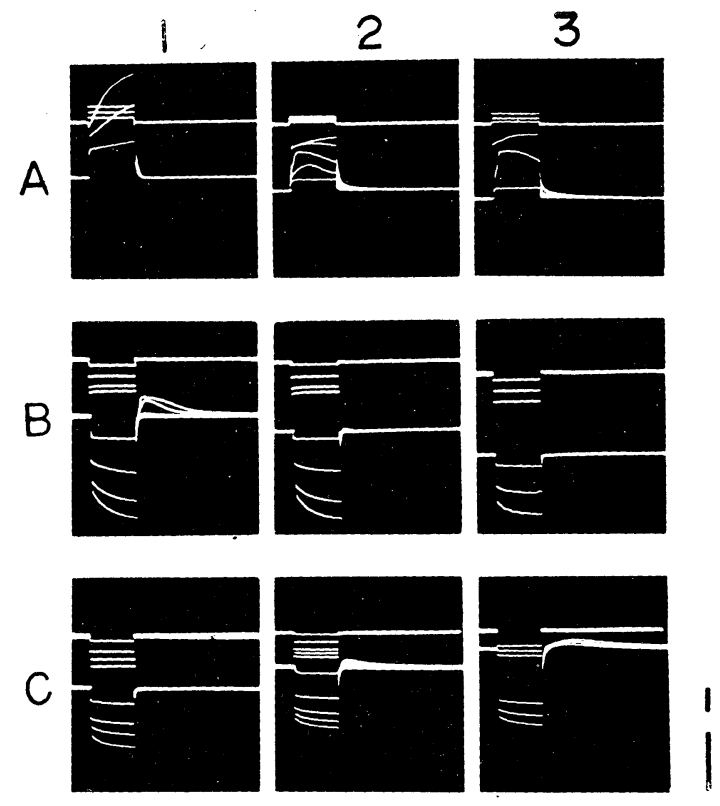

FIg. 10. Records in $12 \mathrm{mM} \mathrm{K}$ ferrocyanide-Ringer's solution. A-2, 3; B-2, 3: With the conditioning hyperpolarization. C-2, 3: With the conditioning depolarization. Calibration: $10^{-7} \mathrm{~A}$ (upper bar), $50 \mathrm{mV}$ (lower bar), $2 \mathrm{sec}$ (horizontal bar). 
polarization remarkable in the fiber in which the slow response was not clear without the conditioning current. As the depolarization became large, the time course became slow and the peak of the response was delayed.

To the outward current pulse, there was no special response without the conditioning hyperpolarization. However when the membrane was hyperpolarized, the slow response was observed in all-or-none manner by the depolarization over a certain critical level as seen in FIG. 10A. This response became larger as the hyperpolarization was increased because the peak of the response showed almost constant level (FIG. 10, A-2, 3). The hyperpolarization being large, the small spike potential happened to be observed in the rising phase.

In order to observe how the membrane resistance was changed after cessation of the current pulse, small pulses were applied to the membrane. FIG. 11-1 shows the result obtained in normal $\mathrm{K}$ concentration and FIG. 11-2, 3, the
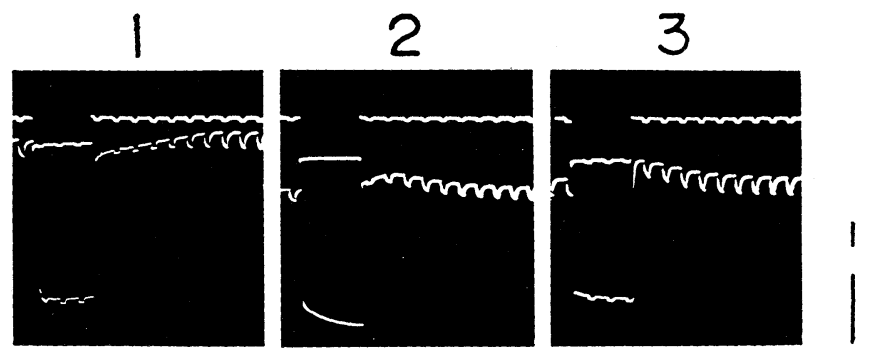

FIG. 11. 1: Record in ferrocyanide-Ringer's solution. 2 and 3 : Records in $12 \mathrm{mM} \mathrm{K}$-ferrocyanide-Ringer's solution. Calibration : $10^{-7} \mathrm{~A}$ (upper bar), $50 \mathrm{mV}$ (lower bar), $2 \mathrm{sec}$ (horizontal bar).

results obtained in $12 \mathrm{mM}-\mathrm{K}$ ferrocyanide-Ringer's solutions. In the process returning slowly to the original potential level in normal $\mathrm{K}$ solution, the resistance was remarkably decreased, while in the process beyond the original potential level in $\mathrm{K}$-excess solution, the resistance was decreased in the rising phase and then it was slightly increased after the peak.

\section{DISCUSSION}

FALK and LANDA (1960b) observed two different distributions of the resting potential in K-free ferrocyanide solution. The low resting potential corresponded to the plateau level of the prolonged action potential which failed to be repolarized in the absence of external K. Two different distributions of the resting potential were observed not only in $\mathrm{Cl}$-free solution without external $\mathrm{K}$, but in sulphate- and glutamate-solutions with normal $\mathrm{K}$ concentration, though the fibers with the low resting potential was increased in number in $\mathrm{K}$-free solutions. 
Since $\mathrm{Cl}$ conductance accounts for two-thirds of the total membrane conductance in the muscle fiber in the resting state (HoDGKIN and Horowicz, 1959; Hutter and Noble, 1960; Adrian and Freygang, 1962), the resting potential is depolarized by the change of $\mathrm{Cl}$ equilibrium potential $\left(\mathrm{E}_{\mathrm{Cl}}\right)$ due to replacement of $\mathrm{Cl}$ with less permeable anion. Then this depolarization of the resting potential increases $\mathrm{Na}$ permeability $\left(\mathrm{P}_{\mathrm{Na}}\right)$ and produces the regenerative depolarization. The presence of $\mathrm{Na}$ in external solution is important for the large depolarization in $\mathrm{Cl}$-free solution, because the depolarization is small in $\mathrm{Na}$ - and $\mathrm{Cl}$-free solution (ADRIAN and FREYGANG, 1962; TOMITA and SzAIMI, 1963). $P_{\mathrm{Na}}$ is, however, decreased by inactivation at this depolarization. $\mathrm{P}_{\mathrm{K}}$ also falls by depolarization because it is related inversely to the size of the outward driving force on potassium (HoDGKIN and HoRowicz, 1959). As the result, the resting potential remains depolarized near to $\mathrm{E}_{\mathrm{Cl}}$. As internal $\mathrm{Cl}$ is decreased, $E_{\mathrm{Cl}}$ approaches $\mathrm{K}$ equilibrium potential $\left(\mathrm{E}_{\mathrm{K}}\right)$ and the resting potential begins to increase again and returns to the original resting potential with increase of $P_{K}$.

The depolarization in $\mathrm{Cl}$-free solution, however, may not be explained only by shift of the membrane potential from $E_{K}$ to $E_{C l}$, because the membrane potential is little depolarized in Na-free solution and it is not dependent on the external $\mathrm{Cl}$ concentration (FALK and LANDA, 1960). Besides, the positive resting potential is not observed in $\mathrm{Cl}$-free solution unless the muscle is loaded with $\mathrm{KCl}$. The depolarization in $\mathrm{Cl}$-free solution might result from some change of the membrane structure due to change of constituent in the external anion. This change might be strengthened by presence of $\mathrm{Na}$ and absence of $\mathrm{K}$ in the external solution.

The fiber with the small resting potential in Cl-free Ringer's solution containing normal $\mathrm{K}$ concentration has much higher membrane resistance than that with the large resting potential, and it has little rectification. The reason why the membrane resistance is high in the depolarized membrane may be due to decrease of $\mathrm{P}_{F}$, and also to slow exit of internal $\mathrm{Cl}$ from the fiber in the absence of external $\mathrm{Cl}$ (HARRIS, 1958). However, it seems rather difficult to explain the decrease of $\mathrm{P}_{\mathrm{K}}$ only by driving force on $\mathrm{K}$ because little rectification is observed in the voltage-current relation obtained by the test pulse with $1 \mathrm{sec}$ duration. The effective membrane resistance was a little decreased though the membrane was hyperpolarized near to the normal resting potential. However, if the test pulse with longer duration were applied, the rectification might be more clearly observed. Anyhow potassium permeability would be not only changed by the driving force but changed slowly with time (ADRIAN, 1960). Presumably the behavior of the membrane in transient displacement of the membrane potential is different from that in the steady state.

The anomalous rectification was more remarkable in $\mathrm{K}$-rich $\mathrm{Cl}$-free solution than in K-rich Cl-Ringer's solution or Cl-free Ringer's solution containing 
normal $\mathrm{K}$ concentration. Though the anomalous rectification in muscle membrane has been explained only by the change of $\mathrm{P}_{\mathrm{K}}$ (HODGKIN and HorowICz, 1959 ; Adrian, 1960 ; NAKAJIMA, IWASAKI and ObATA, 1962), it may be partly explained by the assumption that the outward current is carried mainly by anions and the inward current is carried mainly by cations.

At the membrane depolarized in low-K Cl-free solution, the membrane potential is near to $E_{\mathrm{Cl}}$ and far from $E_{K}$. Being hyperpolarized by the inward current, the membrane potential approaches $E_{K}$, and $P_{K}$ is increased. The membrane potential quickly returns near to the original potential level due to small membrane time constant. However, the increase of $P_{K}$ makes the membrane potential remain near $E_{K}$ rather than the resting state and return gradually to the original potential level with recovery of $P_{K}$. This phenomenon resembles what is observed in the nerve in K-rich solution (TASAKI, 1959; KoKeTsu and Koyama, 1962) but the mechanism may be different in the direction of change of $\mathrm{P}_{\mathrm{K}}$.

When the original resting potential is a little more negative than $E_{\widetilde{c}}$, the membrane potential comes closer to $\mathrm{E}_{\mathrm{Cl}}$ after termination of the test pulse, namely the inside potential becomes more positive than the original level.

When the membrane is hyperpolarized by the conditioning inward current, IR-drop produced by the conditioning current is decreased due to the decrease of the membrane resistance after cessation of the test pulse. This augments the slow depolarization. It is considered that the strong hyperpolarization removes inactivation of $\mathrm{Na}$ conductance, and that the similar phenomenon to the anode break response observed in the node of Ranvier (OOYAMA and WRIGHT, 1961) occurs. Na response is followed by the slow response which might be due to slow change of $\mathrm{P}_{\mathrm{K}}$ and $\mathrm{P}_{\mathrm{Na}}$.

Since the membrane potential in $\mathrm{K}$-rich $\mathrm{Cl}$-free solution nearly accords with $\mathrm{E}_{K}, \mathrm{P}_{K}$ is a little decreased by the hyperpolarization (KOKETSU and NODA, 1962), though it is not so prominent as in the nerve. While the decrease of $P_{K}$ is slowly recovered after cessation of the current pulse, the membrane potential approaches $E_{\leftrightharpoons \jmath}$ which is more positive than $E_{K}$, and it becomes beyond the original level. When it becomes near to $E_{2}, P_{K}$ is much decreased by the anomalous rectification. At the moment the membrane response will be produced by increase of $\mathrm{Na}$ conductance if inactivation of $\mathrm{Na}$ conductance is removed by the hyperpolarization.

In $\mathrm{K}$-rich $\mathrm{Cl}$-free solution, the conditioning inward current works to reduce the change of the membrane potential after cessation of the test pulse, for IR-drop by the conditioning current is oppositely changed to the membrane potential which tends to move toward $\mathrm{E}_{\varsigma}$. The conditioning outward current has the opposite effect to the inward test pulse for the same reason. However, when the membrane potential comes near to $\mathrm{E}_{\mathrm{C}}$ by the conditioning current, the response becomes small again. In this case, the response by $\mathrm{Na}$ can not 
be expected because $\mathrm{Na}$ conductance is almost completely inactivated by the conditioning outward current.

When the inward current is passed through the membrane, the increase of the internal $\mathrm{Cl}$ concentration can be expected by electrophoretic injection of $\mathrm{Cl}$ from a microelectrode filled with $3 \mathrm{M} \mathrm{KCl}$ solution. As the result, the slow break response may be caused by the fact that $E_{\mathrm{Cl}}$ becomes more positive inside.

If the assumption is true, the response should be increased by the conditioning inward current, but actually it was not so. Thus the response is not considered to be due to $\mathrm{Cl}$-injection.

\section{SUMMARY}

1. In Cl-free sulphate-, ferrocyanide- and glutamate-Ringer's solutions, the frog skeletal muscle fiber showed two different distributions of the resting potentials, one of which had the peak at about $-80 \mathrm{mV}$ and the other, about $-30 \mathrm{mV}$. The difference of the resting potentials in two groups was clear in $\mathrm{K}$-free solution.

2. The fiber with the low resting potential had a much higher membrane resistance than that of the fiber with the high resting potential. The low resting potential seemed to correspond to the plateau of the prolonged action potential at which both $\mathrm{Na}$ and $\mathrm{K}$ conductances were inactivated. In such condition, the fiber showed the slow break response after cessation of the strong inward current pulse. The response may be caused by the increase of the $\mathrm{K}$-conductance due to the hyperpolarization. Form of the response was varied depending on the membrane potential level.

3. In $\mathrm{K}$-rich $\mathrm{Cl}$-free solution, the slow break response was also observed after termination of the inward current pulse. This response may be explained by the decrease of the $\mathrm{K}$-conductance due to the hyperpolarization.

We wish to thank Prof. N. ToIDA for his discussion on this work and reading this manuscript.

\section{REFERENCES}

1) Adrian, R.H. Potassium chloride movement and the membrane potential of frog muscle. J. Physiol. 151 : 154-185 (1960).

2) Adrian, R.H. And Freygang, W.H. The potassium and chloride conductance of frog muscle membrane. J. Physiol. 163: 61-103 (1962).

3) Falk, G. And Landa, J.F. Prolonged response of skeletal muscle in the absence of penetrating anions. Am. J. Physiol. 198: 289-299 (1960a).

4) FAlK, G. And Landa, J.F. Effects of potassium on frog skeletal muscle in a chloride-deficient medium. Am. J. Physiol. 198: 1225-1231 (1960b).

5) Harris, E. J. Anion interaction in frog muscle. J. Physiol. 141: 351-365 (1958).

6) Hodgkin, A. L. And Horowicz, P. The influence of potassium and chloride ions 
on the membrane potential of single muscle fibres. J. Physiol. $148: 127-160$ (1959).

7) Hutter, O.F. And Noble, D. The chloride conductance of frog skeletal muscle. J. Physiol. $151:$ 89-102 (1960).

8) Koketsu, K. And Noda, K. Membrane responses of frog skeletal muscle fibers in calcium-free media. J. Cell. Comp. Physiol. 59 : 323-332 (1962).

9) Konetsu, K. And Koyama, I. Membrane responses of frog's spinal ganglion cells in calcium-free solutions. J. Physiol. $163: 1-12$ (1962).

10) Nakajima, S., Iwasaki, S. And Obata, I. Delayed rectification and anomalous rectification in frog's skeletal muscle membrane. J. Gen. Physiol. 46: 97-115 (1962).

11) Ooyama, H. and Wright, E. B. Anode break excitation on single Ranvier node of frog nerve. Am. J. Physiol. 200 : 209-218 (1961).

12) Segal, J. An anodal threshold phenomenon in the squid giant axon. Nature 182: 1370-1372 (1958).

13) Stämpfli, R. Die Strom-Spannungs-Charakteristik der erregbaren Membran eines einzelnen Schnürrings und ihre Abhängigkeit von der Ionen Konzentration. Helv. Physiol. Acta 16:127-145 (1958).

14) TAsaki, I. Demonstration of two stable states of the nerve membrane in potassium-rich media. J. Physiol. 148: 306-331 (1959).

15) Tomita, T. And Szaimi, T. Electrical properties of the frog skeletal muscle membrane in $\mathrm{Na}$ - and Cl-free solutions. Jap. J. Physiol. In preparation. 